\title{
Low GAS5 expression may predict poor survival and cisplatin resistance in cervical cancer
}

\author{
Xingyu Fang ${ }^{1,2}$, Guanglei Zhong1, Yuhan Wang ${ }^{1}$, Zhongqiu Lin ${ }^{1}$, Rongchun Lin ${ }^{1}$ and Tingting Yao ${ }^{1,2}$
}

\begin{abstract}
Cisplatin resistance is a major challenge in cervical cancer (CC) chemotherapy. Growth arrest-specific 5 (GAS5) has been reported to be a tumour suppressor gene in CC. However, the mechanism of GAS5 in chemoresistance remains undetermined. Our research evaluated GAS5 expression in normal and CC tissues by qPCR and in situ hybridization (ISH). Statistical analysis was conducted to analyse the association of GAS5 expression with survival. Biochemical methods were used to screen upstream and downstream regulators of GAS5. Then, interactions were confirmed by ChIP, RNA pull-down, RNA immunoprecipitation (RIP), dual-luciferase reporter and real-time PCR assays. The cisplatin sensitivity of GAS5-overexpressing CC cells was demonstrated in vitro and in vivo. The results showed that low GAS5 expression was correlated with poor overall survival. Mechanistically, GAS5 was transcriptionally modulated by P-STAT3 and served as a competing endogenous RNA (ceRNA) of miR-21 to indirectly affect cisplatin sensitivity through PDCD4 regulation in CC cells. Animal studies confirmed that GAS5 enhanced cisplatin sensitivity and promoted PDCD4 expression in vivo. GAS5 was regulated by P-STAT3 and affected the sensitivity of CC to cisplatin-based chemotherapy through the miR-21/PDCD4 axis. This result may provide new insight into cisplatin-based therapy.
\end{abstract}

\section{Introduction}

Cervical cancer $(\mathrm{CC})$ remains the second leading cause of cancer death in women aged 20-39 years. CC mortality among women in less developed countries is twice that of women in affluent countries ${ }^{1}$. Persistent human papillomavirus (HPV) infection, most commonly with HPV16, is a high-risk factor for $\mathrm{CC}^{2}$. Despite advances in surgical resection, chemoradiotherapy and anti-angiogenic therapy for CC, the long-term survival rate of $\mathrm{CC}$ patients remains unsatisfactory due to metastasis and recurrence ${ }^{3-5}$. Currently, cisplatin (DDP)-based concurrent chemoradiotherapy is the standard treatment for locally advanced $\mathrm{CC}^{6}$. A study found that consolidation chemotherapy after cisplatin-based chemoradiotherapy can control

\footnotetext{
Correspondence: Tingting Yao (yaotting@mail.sysu.edu.cn)

${ }^{1}$ Department of Gynecological Oncology, Sun Yat-sen Memorial Hospital, Sun Yat-sen University, 107 Yan Jiang West Road, 510120 Guangzhou, People's

Republic of China

${ }^{2}$ Guangdong Provincial Key Laboratory of Malignant Tumor Epigenetics and Gene Regulation, Sun Yat-Sen Memorial Hospital, Sun Yat-Sen University,

510120 Guangzhou, China

Edited by A. Stephanou
}

distant relapse and improve overall survival (OS) and progression-free survival ${ }^{7}$. However, cisplatin resistance remains a serious problem during the treatment of $\mathrm{CC}$, which greatly restricts the clinical application and efficacy of cisplatin ${ }^{8}$. Thus, investigating the theory and mechanism underlying the chemosensitivity of $\mathrm{CC}$ cells is an urgent task.

Chemotherapeutic resistance in $\mathrm{CC}$ is usually caused by changes in cellular drug uptake, decreased drug influx and increased drug efflux, drug detoxification via cellular mercaptans, drug target alterations and DNA repair ${ }^{9}$. In addition, hypoxia, epithelial-mesenchymal transition, apoptosis inhibition and changes in molecular signalling pathways also contribute to drug resistance ${ }^{10}$. Studies have suggested that cisplatin resistance is related to regulation of the cell cycle, apoptosis and the Wnt pathway by long non-coding RNAs (lncRNAs) ${ }^{11}$. LncRNAs are small non-coding RNAs with more than 200 nucleotides and account for $80 \%$ of all non-coding RNAs. LncRNAs can regulate gene transcription by binding with chromatin regulatory proteins and via gene silencing, DNA 
methylation, histone modification, hybridization and other mechanisms ${ }^{12}$. Growth arrest-specific 5 (GaS5), located on chromosome 1q25, plays an anticancer role in breast, prostate and ovarian cancers and in other tumours $^{13-15}$. Our previous studies have proven that GAS5 inhibits the proliferation, invasion, migration and apoptosis of $\mathrm{CC}$ cells ${ }^{16}$. However, the functional mechanism and the upstream transcription factor for GAS5 in CC remain unknown.

Recently, a large number of studies have suggested cross-modulation between lncRNAs and microRNAs (miRNAs). LncRNAs bind and sequester miRNAs and block their functions ${ }^{17}$. MiRNAs regulate gene expression by binding to the $3^{\prime}$-untranslated regions (3'-UTRs) of their target genes ${ }^{18}$. In our previous study, miR-21 was identified to be significantly overexpressed in human cervical cell lines and squamous cancer tissues and to regulate the proliferation, apoptosis and migration of HPV16-positive cervical squamous cells ${ }^{19}$. Research found that miR-21 was a target of GAS5 in bladder cancer cells $^{20}$. Therefore, the association of GAS5 and miR-21 in $\mathrm{CC}$ remains uncertain.

PDCD4, a target gene of miR-21, has been considered an efficient suppressive regulator in many cancers, including CC. Overexpression of PDCD4 can inhibit apoptosis and enhance chemosensitivity to cisplatin ${ }^{21,22}$. Research has shown that miR-21 suppression could affect cisplatin sensitivity by mediating the PTEN/PI3K/AKT and PDCD4/JNK signalling pathways in A549/DDP cells $^{23}$. In nasopharyngeal carcinoma, PDCD4 enhances cisplatin sensitivity by stimulating the PI3K/AKT/c-jun feedback loop ${ }^{24}$. However, whether PDCD4 is involved in the influence of GAS5 on cisplatin sensitivity in CC has not been determined.

Our study revealed that GAS5 can bind and sequester miR-21 and further affect the expression of PDCD4, which enhanced cisplatin sensitivity in CC. The transcription factor phosphorylated signal transducer and activator of transcription (P-STAT3) bound to the promoter region of GAS5 to transcriptionally modulate its expression. Thus, the STAT3-regulated GAS5/miR-21-5p/PDCD4 axis may have therapeutic potential for restoring the effectiveness of cisplatin-based chemotherapy in CC.

\section{Materials and methods}

\section{Cell culture and tissue samples}

HeLa and SiHa CC cells and 293T cells (both obtained from the American Type Culture Collection (ATCC)) were grown in Dulbecco's modified Eagle's medium (Gibco; Thermo Fisher Scientific, Inc.) supplemented with $10 \%$ foetal calf serum (Gibco; Thermo Fisher Scientific, Inc.), $100 \mathrm{IU} / \mathrm{ml}$ penicillin $\mathrm{G}$ and $100 \mathrm{mg} / \mathrm{ml}$ streptomycin sulfate (Sigma-Aldrich, St. Louis, MO, USA). Cells were incubated at $37^{\circ} \mathrm{C}$ in a humidified $5 \% \mathrm{CO}_{2}$ atmosphere.
The specimens used for quantitative PCR (qPCR) and for preparation of in situ hybridization (ISH) slides were harvested with informed consent from patients with CC who underwent surgery at Sun Yat-sen Memorial Hospital. The study was approved by the Institutional Review Board of Sun Yat-sen Memorial Hospital. Two independent cohorts were enrolled for this study. Cohort 1 comprised 30 paired CC tissues and adjacent non-tumour tissues obtained from patients during surgery. Cohort 2 comprised 88 paraffin-embedded CC specimens and 19 adjacent normal specimens collected from the Department of Pathology in Sun Yat-sen Memorial Hospital.

\section{Bioinformatic analysis}

TCGA (The Cancer Genome Atlas) expression data for the lncRNA GAS5 in 306 cancer and 13 normal tissue samples were acquired from GEPIA. The expression levels of miR-21, STAT3 and PDCD4 were downloaded from UCSC Xena and SangerBox. All data are available online, and access does not require patient consent or other permissions. The use of the data does not violate the rights of any person or institution.

Potential transcription factors were predicted using the UCSC tool. After screening the target genes, the binding sites in the upstream promoter binding region in GAS5 were predicted with the JASPAR CORE database.

The potential interaction sites in GAS5 and miR-21 were predicted with the web-based online server ViennaRNA Web Services. The interaction between miR-21 and the $3^{\prime}$-UTR of PDCD4 was predicted with the online database miRDB.

\section{Chromatin immunoprecipitation (ChIP) assay}

A SimpleChIP Enzymatic Chromatin IP Kit (CST) was used for the ChIP assay according to the manufacturer's protocol. Briefly, HeLa cells were incubated with $1 \%$ formaldehyde for $10 \mathrm{~min}$ to generate DNA-protein crosslinks. Then, cell lysates were sonicated into $100-500 \mathrm{bp}$ fragments and immunoprecipitated overnight separately with anti-P-STAT3 (\#9145T, Cell Signalling Technology), anti-histone $\mathrm{H} 3$ (the positive control) and anti-rabbit immunoglobulin G (the negative control (NC)) antibodies. After incubation with beads for $2 \mathrm{~h}$, the immunoprecipitated DNA was eluted by incubation in $100 \mu \mathrm{l}$ of ChIP elution buffer containing $10 \mu$ lof proteinase $\mathrm{K}$ at $65^{\circ} \mathrm{C}$ for $30 \mathrm{~min}$ with rotation. The eluted DNA was purified and then dissolved in $50 \mu \mathrm{l}$ of DNA elution buffer. The JASPAR CORE database was used to identify the top four binding sites between STAT3 and the genomic region 2000 bp upstream of the GAS5 sequence. Immunoprecipitated chromatin was analysed by qPCR using primers targeting the predicted binding sites. The primer sequences used for ChIP-qPCR are listed in Supplementary Table 2. 


\section{Construction of plasmid vectors and cell lines stably transfected with GAS5}

The cDNA sequence encoding GAS5 was PCR amplified and then subcloned into the vector pLVX-IRES-Puro. The empty pLVX-IRES-Puro vector was used as the control. Stably transfected cell lines were constructed as described below. HeLa and SiHa CC cells were seeded in a six-well plate. When the cells were $70-80 \%$ confluent, the GAS5 lentiviral vector (constructed by GeneChem, Shanghai, China) and HitransP G were added for infection. Fortyeight hours after lentiviral infection, cells were transferred into $10-\mathrm{cm}$ culture dishes. Puromycin $(4 \mu \mathrm{g} / \mathrm{ml})$ was added to screen stably transfected cells. Cells were harvested 3 days after screening, and quantitative real-time polymerase chain reaction (qRT-PCR) was conducted to evaluate the transfection efficiency.

\section{Transfection}

The miR-21-5p mimic, si-PDCD4 and the corresponding $\mathrm{NC}$ sequences were purchased from GenePharma (Guangzhou, China). Briefly, CC cells were seeded in a six-well plate. When the cells were $70-80 \%$ confluent, the miR-21 inhibitor, miR-21 inhibitor NC, miR-21 mimic, miR-21 mimic NC, or si-PDCD4 at a final concentration of $20 \mathrm{nM}$ was transfected into HeLa or SiHa cells using Lipofectamine $^{\mathrm{TM}} 3000$ (Invitrogen/Life Technologies, Carlsbad, CA, USA) according to the manufacturer's instructions. For the rescue experiment, the miR-21 mimic or NC was transfected into cell lines stably transfected with GAS5. Functional experiments were performed after transfection for $48 \mathrm{~h}$.

\section{RNA isolation and qRT-PCR}

Total RNA was isolated from cell lines using TRIzol reagent (Takara Bio, Inc., Shiga, Japan), and a total of $1 \mu \mathrm{g}$ RNA was then converted into cDNA using Prime Script RT Master Mix (Takara Bio, Inc.) according to the manufacturer's instructions. The specific miR-21-5p reverse transcription primer and the U6 gene reverse transcription primers were added to the miR-21 and U6 RT-PCR samples, respectively. The expression levels of GAS5, PDCD4 and miR-21 were determined by qRT-PCR using a SYBR GREEN MIX kit (Takara Bio, Inc., Shiga, Japan) according to the manufacturer's protocols. $\beta$-Actin was used to normalize the relative expression levels of GAS5 and PDCD4. U6 was used to normalize the relative expression level of miR-21. Fold changes in the relative expression levels of the target genes were calculated using the $2^{-\Delta \Delta C T}$ method. All primers used are listed in Supplementary Table 1.

\section{RNA immunoprecipitation (RIP) assay}

To prove that GAS5 regulated miR-21 as a competitive endogenous RNA (ceRNA), we used the Ago2-LincK RIP assay to detect the association of GAS5 with the RISC complex. Briefly, beads conjugated to an anti-AGO2 antibody (\#ab557113, Abcam, Cambridge, MA, USA) or NC mouse IgG (Millipore, Bedford, MA, USA) were incubated at $4{ }^{\circ} \mathrm{C}$ overnight. Then, more than $10^{7} \mathrm{HeLa}$ cells were harvested in PL buffer $(100 \mathrm{mM} \mathrm{KCl}, 5 \mathrm{mM}$ $\mathrm{MgCl}_{2}, 10 \mathrm{mM}$ HEPES ( $\mathrm{pH}$ 7.0), $400 \mu \mathrm{M}$ VRC, $1 \mathrm{mM}$ DTT and 0.5\% NP-40) containing $100 \mathrm{U} / \mathrm{ml}$ RNase and protease inhibitors, and $100 \mu \mathrm{l}$ of cell lysate was incubated with magnetic beads and the antibodies described above in NT2 buffer (50 mM Tris- $\mathrm{HCl}(\mathrm{pH} 7.4), 150 \mathrm{mM} \mathrm{NaCl}$, $1 \mathrm{mM} \mathrm{MgCl} 2,0.05 \%$ NP-40). Subsequently, the beads were washed five times with cold NT2 buffer and incubated with proteinase $\mathrm{K}$ to digest proteins. RNA associated with the beads was extracted by TRIzol reagent (Takara Bio, Inc., Shiga, Japan) and detected by qRT-PCR. The miR-21 and GAS5 levels were detected by qRT-PCR.

\section{RNA pull-down assay}

RNA pull-down assays were performed to prove the interaction between GAS5 and miR-21. Full-length GAS5 was transcribed in vitro with T7 RNA Pol II (Promega, Madison, WI) and labelled with biotin using Biotin RNA Labeling Mix (Roche, USA). An oligo probe (Bio-NC) was used as the NC. HeLa cells were fixed with formaldehyde to cross-link RNA and then suspended in $1 \mathrm{ml}$ of lysis buffer $(20 \mathrm{mM}$ Tris- $\mathrm{HCl}, 200 \mathrm{mM} \mathrm{NaCl}$, $2.5 \mathrm{mM} \mathrm{MgCl}_{2} \cdot 6 \mathrm{H}_{2} \mathrm{O}, 0.05 \%$ IGEPAL) containing RNase and protease inhibitors. Three micrograms of biotinylated RNA (Bio-GAS5, Bio-NC) was incubated upside down with Streptavidin Magnetic Beads (\#88816, Invitrogen) at room temperature for $2 \mathrm{~h}$. Then, we added $500 \mu \mathrm{l}$ of cell lysate supernatant to the magnetic beads for another $2 \mathrm{~h}$ of incubation at $4{ }^{\circ} \mathrm{C}$. After sequential elution with solution A (100 mM NaOH, $50 \mathrm{mM} \mathrm{NaCl})$ and solution $\mathrm{B}(100 \mathrm{mM} \mathrm{NaCl})$ three times, the magnetic beads were resuspended in $200 \mu \mathrm{l}$ of lysis buffer and rotated at room temperature for $2 \mathrm{~h}$ to release the formaldehyde cross-linked RNA in the sample. The RNA-RNA complexes were purified using TRIzol reagent (Takara). The miR-21 level in the RNA-RNA complexes was detected by qRT-PCR.

\section{Dual-luciferase reporter assay}

The wild-type (WT) PDCD4 3'-UTR fragment was cloned downstream of the Renilla luciferase gene in the psiCHECK-2 vector (Promega, Madison, WI). Mutants were generated by mutating base pairs in the miR-21binding sequences. WT miR-21 with GAS5-binding sequences and mutant-type (MUT) miR-21 with no GAS5-binding sequences were cloned using the same procedure. To study the relationship between GAS5 and miR-21, we used Lipofectamine 3000 (Thermo Fisher Scientific, Inc.) to co-transfect the WT or MUT miR-21 
luciferase reporter with a GAS5 overexpression plasmid or its control vector into 293T cells. To clarify the targeting relationship between miR-21 and PDCD4, we cotransfected the WT or MUT-PDCD4 3'-UTR luciferase reporter plasmid with the miR-21 mimic or the corresponding NC into 293T cells. Cells were harvested $48 \mathrm{~h}$ after transfection, and Renilla and firefly luciferase activities were analysed using the Dual-Luciferase ${ }^{\circledR}$ Reporter Assay System (\#E2920, Promega). Renilla and Firefly luciferase activity values were normalized to the firefly luciferase activity value for each transfected well. All assays were performed in triplicate.

\section{Detection of apoptosis}

After transfection for $48 \mathrm{~h}$, SiHa cells were treated with $15 \mu \mathrm{g} / \mathrm{ml}$ cisplatin for $48 \mathrm{~h}$. The cells were then digested with EDTA-free trypsin and washed twice with cold phosphate-buffered saline (PBS). After incubation with Annexin-V and PI for 10 min at room temperature, flow cytometry was performed using a flow cytometer (FACSCalibur, Becton Dickinson, Franklin Lakes, NJ, USA).

\section{Western blot analysis}

After treatment, cells were harvested and lysed in icecold RIPA lysis buffer (FD, Hangzhou, China) containing a protease inhibitor cocktail and phosphatase inhibitor cocktail on ice for $30 \mathrm{~min}$. After centrifugation at $13,000 \times g$ for $30 \mathrm{~min}$ at $4{ }^{\circ} \mathrm{C}$, the protein content in the supernatant was quantified using a BCA protein assay kit (CWBIO, Beijing, China). The protein samples were then heated at $100^{\circ} \mathrm{C}$ for $10 \mathrm{~min}$, and equal amounts $(30 \mu \mathrm{g})$ of protein were separated by 8 and 12\% sodium dodecyl sulfate denaturing polyacrylamide gel electrophoresis and transferred onto $0.22-\mu \mathrm{m}$ polyvinylidene difluoride membranes (Millipore, Bedford, MA, USA). The membranes were blocked with $5 \%$ non-fat milk for $1 \mathrm{~h}$ at room temperature and then incubated first with rabbit antihuman antibodies specific for PDCD4 (1:2000 dilution; Cell Signalling Technology), PARP (1:1000 dilution; Cell Signalling Technology), caspase-3 (1:1000 dilution; Cell Signalling Technology), STAT3 (1:1000 dilution; Cell Signalling Technology) and P-STAT3 (1:1000 dilution; Cell Signalling Technology) at the recommended dilutions and with mouse anti-human antibodies specific for GAPDH (1:5000 dilution; FD) overnight at $4{ }^{\circ} \mathrm{C}$ and then with horseradish peroxidase (HRP)-conjugated goat anti-rabbit $\operatorname{IgG}(1: 10,000)$ or goat anti-mouse $\operatorname{IgG}$ $(1: 10,000)$ for $2 \mathrm{~h}$ at $37^{\circ} \mathrm{C}$. After the membranes were washed in PBS containing Tween 20, signals were visualized with an ECL kit (Millipore, USA) and analysed using a FluorChem FC2 Imaging System (Alpha Innotech, San Leandro, CA, USA). The protein expression levels were normalized to those of GAPDH as the internal control.

\section{In situ hybridization}

The expression level of GAS5 in tissues was measured with digoxigenin-labelled antisense oligonucleotide probes. Slides of $\mathrm{CC}$ tissue were dewaxed and rehydrated. After treatment with $3 \% \mathrm{H}_{2} \mathrm{O}_{2}$ at room temperature for $10 \mathrm{~min}$, tissue slices were digested with pepsin diluted with $3 \%$ citric acid to expose RNA. After incubation with prehybridization buffer for $2 \mathrm{~h}$ at $37^{\circ} \mathrm{C}$, the slices were hybridized with the GAS5 probe (Genebio, Guangzhou, China; $1 \mu \mathrm{M}$ ) at $37^{\circ} \mathrm{C}$ overnight. Sections were then washed with a gradient-diluted SSC solution at $37^{\circ} \mathrm{C}$ for $15 \mathrm{~min}$ and subsequently incubated with biotinylated mouse anti-digoxigenin antibodies for $60 \mathrm{~min}$ at $37^{\circ} \mathrm{C}$. After incubation with both SABC and biotinylated peroxidase at $37^{\circ} \mathrm{C}$ for $20 \mathrm{~min}$, hybridization signals were visualized with $3,3^{\prime}$-diaminobenzidine (DAB) chromogenic substrate (Dako). The reaction was stopped by washing with distilled water for $5 \mathrm{~min}$. Slides were counterstained with haematoxylin, dehydrated with ethyl alcohol, sealed with neutral resins and imaged. The GAS5 probe sequence was as follows: $5^{\prime}$ CCATAAGGTGCTATCCAGAGC- $3^{\prime}$. Two pathologists evaluated the ISH scores in a blinded manner. The staining intensity of GAS5 was scored on a scale of $0-3$ as follows: 0 (negative), 1 (weak), 2 (moderate) and 3 (strong). For statistical analysis, we divided the samples into two groups: slides with scores of 2 and 3 were classified into the high GAS5 expression group and those with scores of 0 and 1 were classified into the lowexpression group.

\section{Xenograft assay in nude mice}

Six female BALB/c nude mice (5 weeks old) were obtained from the Guangdong Medical Laboratory Animal Center and raised in the Department of Laboratory Animal Science, Sun Yat-sen University (Guangzhou, China). All animal experiments were conducted in accordance with the Declaration of Helsinki and were approved by the Research Animal Resource Center of Sun Yat-Sen University (application number 2019000253). Mice were randomly assigned to two groups, with three mice in each group. To establish a subcutaneous model, SiHa cells with GAS5 overexpression and corresponding control cells (stably transduced with lentiviral vectors) were resuspended in PBS at a concentration of $10^{7}$ cells/ $100 \mu \mathrm{l}$. Mice were then subcutaneously injected in the right flank with $100 \mu \mathrm{l}$ of cell suspension to induce tumour formation. When the tumour sizes reached $50 \mathrm{~mm}^{3}$, the mice were injected weekly with $2 \mathrm{mg} / \mathrm{kg}$ cisplatin. Two weeks after the first injection, the mice were killed, and the tumours were excised. Tumour tissues were harvested for tissue processing. A 4-mm portion of each tumour was fixed with paraffin, and immunohistochemistry (IHC) was performed to detect 
proteins. Tumour sizes were measured weekly, and the volumes (in cubic millimetres) were calculated according to the following equation: width ${ }^{2} \times$ length $\times 0.5$. Length and width refer to the longest and shortest diameters, respectively.

\section{Immunohistochemistry}

The protein expression levels of PDCD4 in subcutaneous xenograft mouse tumours were assessed by IHC with the corresponding anti-PDCD4 antibody (1:200 dilution; \#9535, Cell Signalling Technology). The protein expression levels of P-STAT3 in CC tissues were assessed by IHC with the corresponding anti-PSTAT3 antibody (1:100 dilution; \#4113S, Cell Signalling Technology). The tumours were fixed, embedded in paraffin and sectioned into $4-\mu \mathrm{m}$-thick sections. After deparaffinization and rehydration, the sections were incubated with $3 \% \mathrm{H}_{2} \mathrm{O}_{2}$ for $10 \mathrm{~min}$ and then with $10 \%$ normal goat serum for $15 \mathrm{~min}$ at room temperature to block endogenous peroxidase activity and non-specific antigen binding. Histological sections were incubated with primary antibodies at $4{ }^{\circ} \mathrm{C}$ overnight and then rinsed three times with PBS. The sections were incubated with an HRP-conjugated secondary antibody for $30 \mathrm{~min}$ at room temperature. To visualize staining, slides were incubated in Tris- $\mathrm{HCl}$ buffer containing DAB and $0.1 \% \mathrm{H}_{2} \mathrm{O}_{2}$ and then counterstained with haematoxylin.

\section{Subcellular fractionation}

Nuclear and cytosolic fractions were separated using a PARIS $^{\mathrm{TM}}$ Kit (\#AM1921, Thermo Scientific) based on the supplier's recommendation. One billion HeLa and SiHa cells were collected for one test. RNA was extracted, and qRT-PCR was performed to assess the GAS5 levels in the nuclear and cytoplasmic fractions with normalization to MALAT1 (nuclear control) and $\beta$-actin (cytoplasmic control).

\section{Statistical analysis}

Data are presented as the mean values \pm standard deviations (means \pm SDs) in this study. GraphPad Prism 7.0 (La Jolla, CA) and SPSS 20.0 (SPSS, USA) were used to conduct statistical analyses. Student's $t$-test was used to analyse gene expression differences. Multiple comparisons were conducted by one-way ANOVA. Estimation of survival differences was performed using the Kaplan-Meier method and log-rank test. Univariate and multivariate Cox regression analyses were used to identify potential prognosis-associated factors. A chi-square test was performed to analyse clinicopathological characteristics. The Pearson correlation coefficient was used to evaluate correlations. $P$ values less than 0.05 were considered statistically significant.

\section{Results}

\section{GAS5 expression is downregulated in cervical tissues}

To determine the enrichment of GAS5 in CC, we investigated the expression level of GAS5 in the TCGA database via GEPIA, which indicated a low level of GAS5 in cancer tissues compared to adjacent normal tissues (Fig. 1a). To confirm this result, we examined the expression level of GAS5 in $30 \mathrm{CC}$ tissues and adjacent noncancerous tissues (cohort 1). The qPCR results revealed that GAS5 was expressed at low levels in tumour tissues compared to peritumoral tissues (Fig. 1b). In addition, we designed and synthesized ISH probes for GAS5 and used ISH to examine GAS5 expression in 88 $\mathrm{CC}$ tissues and 19 peritumoral tissues (cohort 2). GAS5 staining was observed mainly in the cytoplasm. Consistent with the qRT-PCR results, the expression of GAS5 was reduced in most tumour tissues compared to peritumoral tissues in cervical squamous carcinoma tissues (Fig. 1c) and cervical adenocarcinoma tissues (Fig. 1d). Moreover, the degree of GAS5 expression differed among CC specimens (Fig. 1e). After grouping according to the scores, we further summarized the relationships between GAS5 expression and the clinicopathological features of CC patients, as shown in Table 1. GAS5 expression was significantly correlated with vaginal recurrence or metastasis $(P<0.01)$, chemotherapy $(P<0.01)$ and age $(P<0.05)$. Importantly, Kaplan-Meier analysis illustrated that low GAS5 expression was correlated with poor OS (Fig. 1f) $(P=0.008$, hazard ratio $(\mathrm{HR})=4.775)$. Univariate analysis indicated that GAS5 $(\mathrm{HR}=4.755,95 \%$ confidence interval $(\mathrm{CI})=$ [1.340-16.875], $P=0.016)$ was a potent independent prognostic indicator for $\mathrm{CC}$ patients. However, the $P$ values in multivariate analyses indicated no statistical significance (Table 2). Logistic regression analysis showed that compared to high GAS5 expression, low GAS5 expression increased the risk of recurrence or metastasis, which accounted for the above result (Supplementary Table 3). In summary, we confirmed that GAS5 was connected with prognosis and chemotherapeutic efficacy in CC.

\section{GAS5 is transcriptionally regulated by P-STAT3 in CC}

Inhibition of STAT3 is reported to sensitize tumour cells to cisplatin-induced apoptosis ${ }^{25}$. Immunohistochemical found that P-STAT3 expression was higher in $\mathrm{CC}$ tissues than that in adjacent normal tissues (Fig. 2a). TCGA database analysis showed that STAT3 expression was negatively correlated with GAS5 expression in CC tissues (Fig. 2b). In addition, the expression level of P-STAT3 was higher in CC cells than in normal cervical cells, whereas the total STAT3 level did not differ (Fig. 2c). As STAT3 enters the nucleus after phosphorylation for transcriptional regulation, we sought to 
A

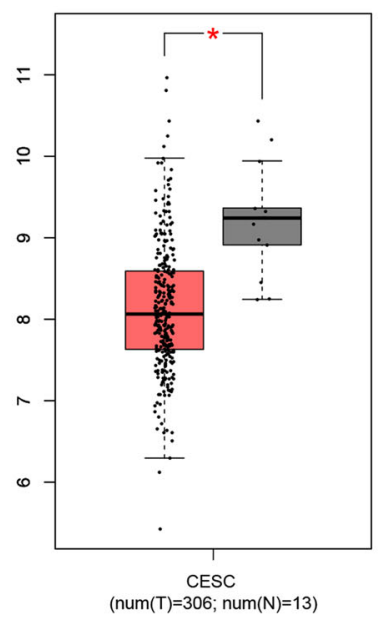

C

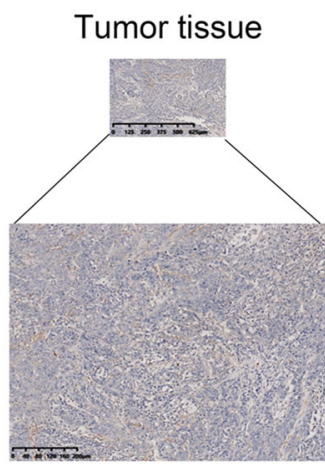

E

Score 0
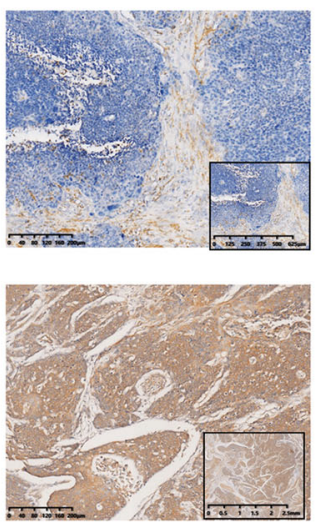

Score 2
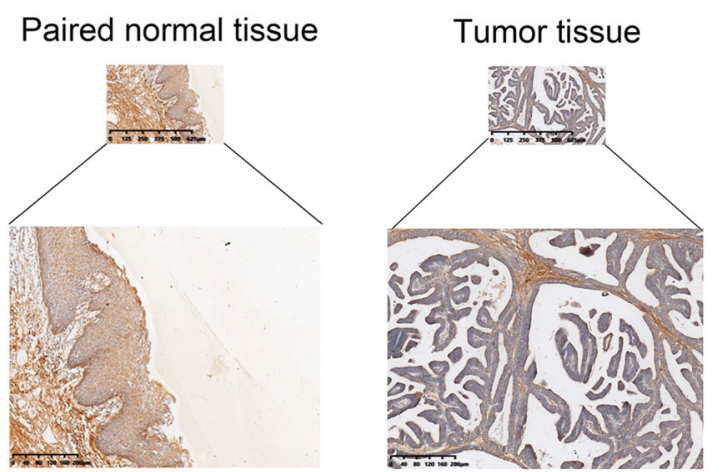

F

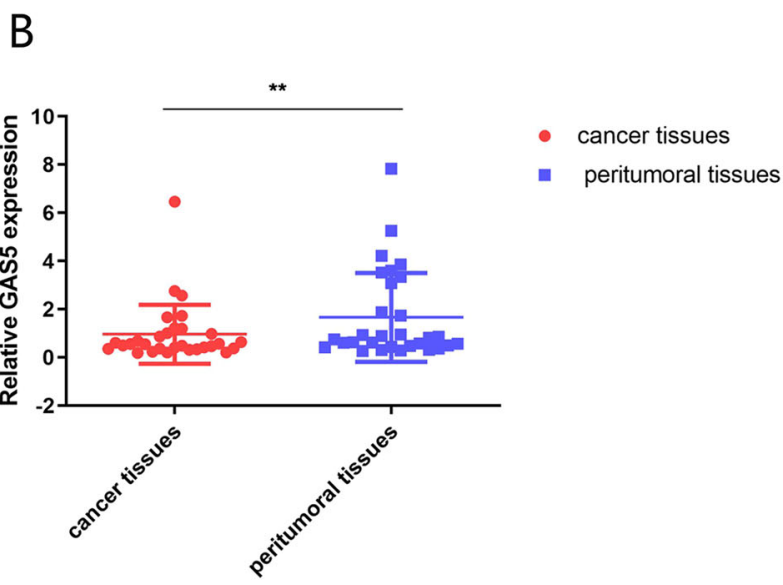

D

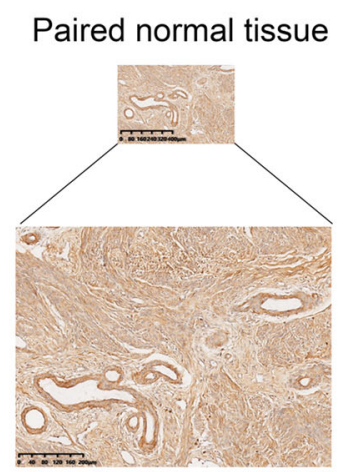

Score 1
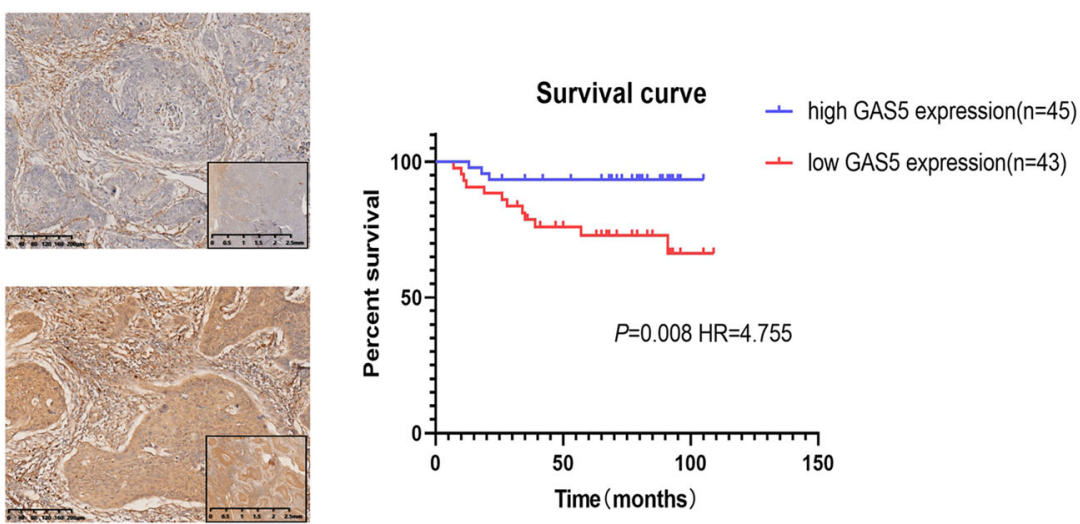

Score 3

Fig. 1 GAS5 is notably downregulated in CC tissues and cell lines. a The expression level of GAS5 in the TCGA database analysed via GEPIA. b GAS5 was downregulated in CC tissues compared to corresponding normal tissues as detected by qPCR in cohort 1. c Representative images of GAS5 expression in cervical squamous carcinoma tissues and adjacent normal tissues as detected by ISH. $\mathbf{d}$ Representative images of GAS5 expression in cervical adenocarcinoma tissues and adjacent normal tissues as detected by ISH. e Representative GAS5 staining patterns in cohort 2. f Low GAS5 expression was correlated with poor overall survival in cervical cancer. 
Table 1 Association of GAS5 expression with clinicopathological features from CC patients.

\begin{tabular}{|c|c|c|c|c|}
\hline Features & $n$ & High & Low & $P$ \\
\hline \multicolumn{5}{|l|}{ Age(years) } \\
\hline$\geq 50$ & 32 & 21 & 11 & $0.048^{*}$ \\
\hline$<50$ & 56 & 24 & 32 & \\
\hline \multicolumn{5}{|l|}{ FIGO stage } \\
\hline | and || & 19 & 7 & 12 & 0.199 \\
\hline III and IV & 69 & 38 & 31 & \\
\hline \multicolumn{5}{|l|}{ Tumour sizes } \\
\hline$\geq 3$ & 38 & 18 & 20 & 0.667 \\
\hline$<3 \mathrm{~F}$ & 50 & 27 & 23 & \\
\hline \multicolumn{5}{|l|}{ LN status } \\
\hline Positive & 20 & 7 & 13 & 0.129 \\
\hline Negative & 68 & 38 & 30 & \\
\hline \multicolumn{5}{|l|}{ Histological grade } \\
\hline Low differentiation & 30 & 15 & 15 & 0.878 \\
\hline Moderate and high differentiation & 58 & 30 & 28 & \\
\hline \multicolumn{5}{|l|}{ Vaginal recurrence or metastasis } \\
\hline Present & 35 & 12 & 23 & $0.016^{*}$ \\
\hline Absent & 53 & 33 & 20 & \\
\hline \multicolumn{5}{|l|}{ Chemotherapy } \\
\hline Yes & 43 & 12 & 31 & $0.001^{*}$ \\
\hline No & 45 & 33 & 12 & \\
\hline
\end{tabular}

determine whether the reduction of GAS5 was related to the phosphorylation of STAT3. Exploring the probable mechanisms using genome bioinformatic analysis, we found that STAT3 was a potential transcription factor for GAS5 (Fig. 2d). We obtained the binding motif of STAT3 from JASPAR and selected four binding sequences for a ChIP experiment (Fig. 2e). As shown in Fig. 2f, P-STAT3 was enriched at site 1 in the promoter region of the GAS5 gene in HeLa cells. To further evaluate the effect of PSTAT3 on GAS5 expression, we inhibited STAT3 phosphorylation with Stattic (Selleck Chemicals, America) at a dose of at least $5 \mathrm{nM}$ in HeLa cells for $36 \mathrm{~h}$ and in $\mathrm{SiHa}$ cells for $24 \mathrm{~h}$. Western blot analysis showed that for HeLa cells, the decline in P-STAT3 exhibited a dose-dependent tendency at concentrations of $50-1000 \mathrm{nM}$, whereas the total STAT3 level remained unchanged. However, at 2000-4000 nM concentration, a simultaneous decline in total STAT3 was observed. For SiHa cells, the dose for a decline in P-STAT3 and no change in total STAT3 was 5-50 nM. Simultaneous decline in total STAT3 was observed from $75 \mathrm{nM}$. (Fig. 2g) Thus, we selected
$1000 \mathrm{nM}$ Stattic for HeLa cells and $50 \mathrm{nM}$ Stattic for SiHa cells to evaluate the effect of STAT3 phosphorylation on GAS5 expression. After adding Stattic, we extracted RNA at $0,6,12,24,36,48$ and $72 \mathrm{~h}$. The qPCR results showed that GAS5 expression was gradually upregulated after $36 \mathrm{~h}$ for HeLa and $24 \mathrm{~h}$ for SiHa (Fig. 2h).

\section{GAS5 is localized mainly in the cytoplasm and acts as a ceRNA in CC by sponging miR-21}

Recently, several mechanisms by which lncRNAs regulate transcription have been reported.

By evaluating the subcellular distribution of GAS5, we found that GAS5 was localized mainly in the cytoplasm (Fig. 3a). Considering the role of miR-21, we hypothesized that GAS5 exerted its function by competitively binding with miR-21 as a ceRNA. We predicted the potential binding sites of miR-21 with GAS5 through ViennaRNA Web Services (Fig. 3b). The secondary structure of GAS5 is shown in Fig. 3c. A dual-luciferase reporter assay was used to verify the binding sites between GAS5 and miR21. GAS5 overexpression markedly attenuated the luciferase reporter activity of WT miR-21 (Fig. 3d).

In addition, we performed an RIP assay using an antiAGO2 antibody to further confirm this effect. GAS5, miR21 and PDCD4 could bind to AGO2, a key component of the RISC complex (Fig. 3e), indicating that GAS5 or PDCD4 formed an RISC complex with miR-21. Subsequently, we used in vitro transcribed biotin-labelled GAS5 to pull-down endogenous miRNAs in HeLa cells and found that miR-21 was significantly enriched compared to biotin-labelled RNA oligo (Fig. 3f). These results demonstrated that GAS5 was physically associated with miR-21 and might function as a ceRNA to affect the target gene of miR-21.

Moreover, rescue experiments were performed to further verify these effects. We used miR-21 mimics to upregulate miR-21 in HeLa and SiHa cell lines stably transfected with GAS5. The expression levels of miR21 and GAS5 were measured by qRT-PCR. GAS5 overexpression was abrogated by miR-21 mimics, and GAS5 also downregulated the expression of miR-21 (Fig. 3g).

\section{PDCD4 is a direct target gene of miR-21}

Through the RIP assay, we discovered that PDCD4 existed in the RISC complex. Then, we revealed a site for miR-21 binding with the $3^{\prime}$-UTR of PDCD4 by miRDB analysis (Fig. 4a). To further verify this site, we inserted fragments of the PDCD4 3'-UTR containing WT or MT miR-21-binding sites into the psiCHECK-2 vector and cotransfected them with the miR-21 mimic. The miR-21 mimic obviously attenuated the relative luciferase activity of the WT PDCD4 reporter, while the MT PDCD4 reporter was unaffected (Fig. 4b). To confirm whether 
Table 2 Univariate and multivariate Cox regression analysis for OS in CC patient.

\begin{tabular}{|c|c|c|c|}
\hline \multirow[t]{2}{*}{ Variable } & \multicolumn{3}{|c|}{ Univariate analysis } \\
\hline & HR & $95 \% \mathrm{Cl}$ & $P$ value \\
\hline \multicolumn{4}{|l|}{ Univariate analyses } \\
\hline Age $(<50$ vs $\geq 50)$ & 1.989 & $0.633-6.252$ & 0.239 \\
\hline FIGO stage (I and II vs III and IV) & 2.303 & $0.819-6.471$ & 0.114 \\
\hline Tumour sizes $\geq 3$ vs $<3$ & 2.835 & $0.968-8.304$ & 0.057 \\
\hline LN status (positive vs negative) & 0.54 & $0.184-1.581$ & 0.261 \\
\hline Histological grade (low differentiation vs moderate and high differentiation) & 0.36 & $0.143-0.908$ & $0.031^{*}$ \\
\hline Vaginal recurrence or metastasis (present vs absent) & 33.266 & $4.313-256.574$ & $0.001^{*}$ \\
\hline GAS5 expression (high vs low) & 4.755 & $1.340-16.875$ & $0.016^{*}$ \\
\hline \multicolumn{4}{|l|}{ Multivariate analyses } \\
\hline Vaginal recurrence or metastasis (present vs absent) & 34.481 & $4.438-267.891$ & 0.001 \\
\hline Histological grade (low differentiation vs moderate and high differentiation) & 0.287 & $0.101-0.816$ & 0.019 \\
\hline GAS5 expression (high vs low) & & & 0.175 \\
\hline
\end{tabular}

$H R$ hazard ratio, $\mathrm{Cl}$ confidence interval.

${ }^{*} P<0.05$.

PDCD4 is a target gene of miR-21, we measured PDCD4 expression in HeLa and SiHa cells after transfection with the miR-21 mimic or inhibitor (Fig. 4c). PDCD4 expression was significantly downregulated in miR-21 mimictreated cells compared to NC-treated cells and markedly upregulated after transfection with the miR-21 inhibitor compared with the NC inhibitor (Fig. 4d).

MiR-21-5p reverses the influence of GAS5 on apoptosis in CC through the PDCD4 pathway

We confirmed that GAS5 promoted apoptosis in CC in our previous research. Flow cytometric analysis revealed that overexpression of GAS5 dramatically increased the apoptosis rate in GAS5 stably transfected SiHa cells, while transfection with the miR-21 mimic markedly reversed this effect (Fig. 5a). We therefore hypothesized that GAS5 regulated apoptosis via miR-21-5p in CC cells.

PDCD4, a target gene of miR-21, is related to apoptosis. We silenced PDCD4 and then determined the apoptosis rate through flow cytometric analysis. Moreover, the apoptosisrelated proteins cleaved PARP and cleaved caspase-3 were also evaluated. The transfection efficiency results demonstrated that si-PDCD4 induced a significant decrease in PDCD4 expression (Fig. 5b). The flow cytometry results indicated that silencing PDCD4 reduced the apoptosis rate in $\mathrm{SiHa}$ cells (Fig. 5c). In order to detect the apoptosis by western blot, we treated cells with $15 \mu \mathrm{g} / \mathrm{ml}$ cisplatin ( $\mathrm{SiHa}$ cells) or $6.5 \mu \mathrm{g} / \mathrm{ml}$ cisplatin (HeLa cells) for $48 \mathrm{~h}$ after transfection for $48 \mathrm{~h}$. Analysis of protein expression showed that the levels of cleaved caspase- 3 and cleaved PARP were reduced after PDCD4 silencing (Fig. 5d).
Based on the above results, we further investigated whether GAS5 regulated cell apoptosis through the PDCD4 pathway and whether cell apoptosis induced by GAS5 expression could be rescued by the miR-21 mimic. Western blotting showed that the increase of PDCD4 in CC cells stably overexpressing GAS5 was restored by the miR-21 mimic, and after cisplatin treatment, the increases of cleaved caspase- 3 and cleaved PARP presented the same trends (Fig. 5e).

These results indicated that GAS5 probably promoted apoptosis in CC by competitively binding to miR-21 and indirectly regulating the PDCD4 pathway.

\section{Expression of PDCD4 in CC tissues and the connection among GAS5, miR-21 and PDCD4}

To validate the above results, we examined the expression levels of miR-21 and PDCD4 in cohort 1 by qPCR. In contrast to GAS5, miR-21 was highly expressed in tumour tissues compared to peritumoral tissues (Fig. 6a), while the trend in PDCD4 expression was the same as that in GAS5 expression (Fig. 6b). TCGA database analysis further confirmed our results. We investigated the PDCD4 expression level in the GEPIA database and the miR-21-5p expression level in GEDS, which showed a low level of PDCD4 and a high level of miR-21-5p in cancer tissues compared with normal tissues (Fig. 6c). Pearson rank correlation analysis of the relationship between GAS5 expression and miR-21 expression in CC patients in the TCGA database indicated a significant negative correlation, while the relationship between GAS5 expression and PDCD4 


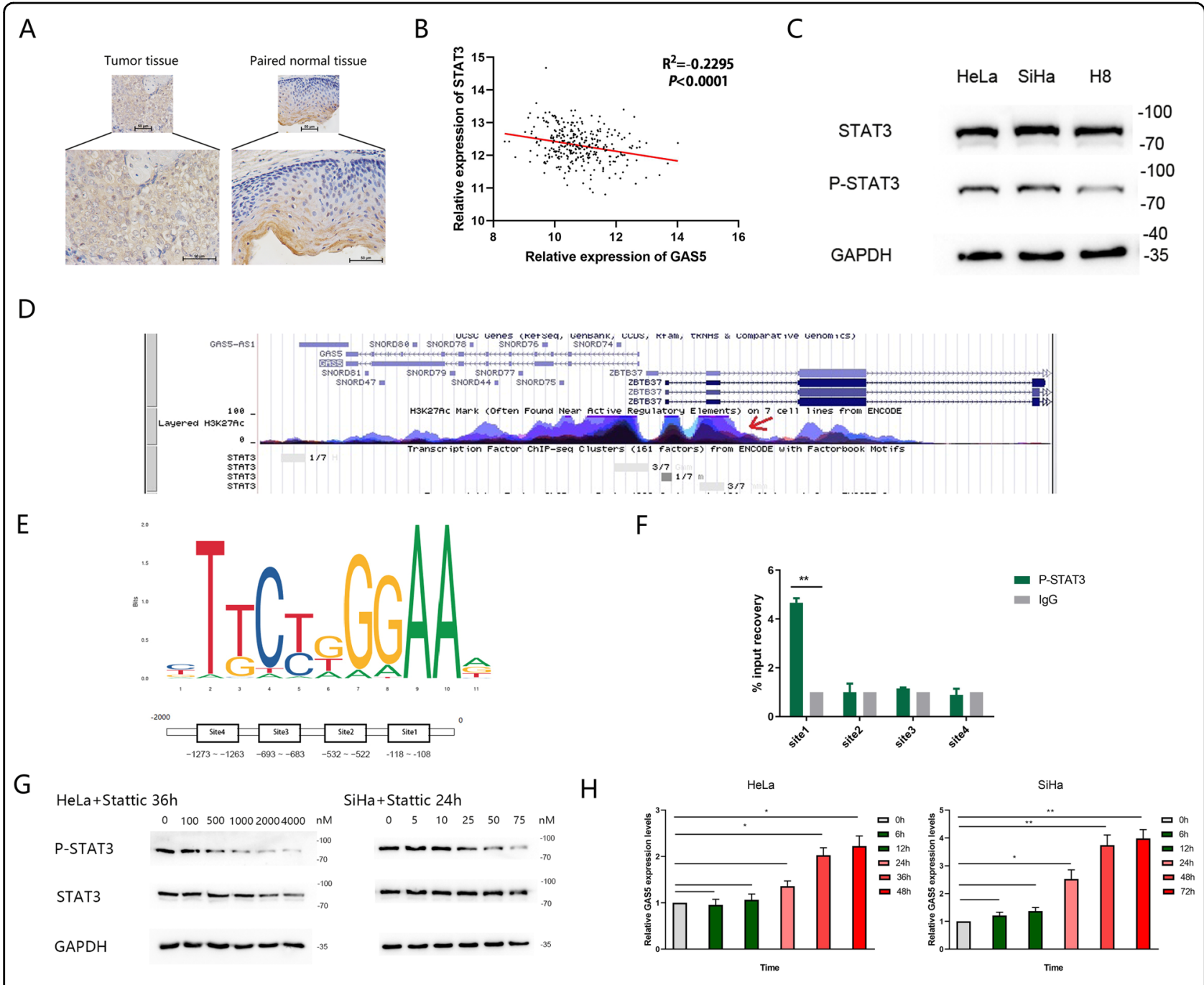

Fig. 2 P-STAT3 binds with the promoter of GAS5 and modulates its transcriptional level. a Immunohistochemical staining for P-STAT3 in cervical cancer tissues and adjacent normal tissues (magnification, $\times 200$ ). Scale bar, $50 \mu \mathrm{m}$. b The correlation between STAT3 and GAS5 expression in CC tissues from the TCGA database was analysed by Pearson correlation analysis. c Western blot analysis showed that the expression levels of P-STAT3 in CC cells were higher than those in normal cervical cell, whereas the total STAT3 level did not differ. $\mathbf{d}$ UCSC database analysis revealed that the promoter of GAS5 had high enrichment of STAT3. e The binding motif of the transcription factor STAT3 was predicted via the JASPAR CORE database. f A ChIP assay demonstrated P-STAT3 binding to site 1 ( -118 to -108$)$ in the GAS5 promoter region. ${ }^{* *} P<0.01$ (Student's t-test). $\mathbf{g}$ Western blot analysis showed that the P-STAT3 level changed dose-dependently under treatment with Stattic, whereas the total STAT3 level remained unchanged at Stattic concentrations of 0-50 nM for SiHa cells and 0-1000 nM for HeLa cells. GAPDH was used as the control. $\mathbf{h}$ A decline in the P-STAT3 level was accompanied by an increase in the GAS5 level. Total RNA was isolated from SiHa cells treated with 50 nM Stattic and HeLa cells treated with $1000 \mathrm{nM}$ Stattic for different durations. GAS5 levels were examined by qRT-PCR. $\beta$-Actin was used as the input control. The fold change was calculated with respect to the control by the $2^{-\Delta \Delta \mathrm{Ct}}$ method. The data are shown as the means \pm SDs $(n=3)$. ${ }^{*} P$ value $<0.05$ compared to untreated cells.

expression indicated a positive correlation (Supplementary Table 4). In addition, a negative correlation was observed for the relationship between GAS5 expression and miR-21 expression while a positive correlation was observed between GAS5 expression and PDCD4 expression $(P<0.05)$ in CC patients of cohort 1 (Fig. 6d). In summary, GAS5 expression was negatively correlated with miR-21 expression and positively associated with PDCD4 expression.

\section{GAS5 enhances the cisplatin sensitivity of CC in vivo}

To further investigate the function of GAS5 in mediating the cisplatin sensitivity of $\mathrm{CC}$ in vivo, GAS5 lentivirus-transfected cells were injected into BALB/c nude mice to establish a xenograft model. One million $\mathrm{SiHa}$ cells were injected subcutaneously into the backs of nude mice, and tumours formed in most mice 4 weeks after cell injection. At the fifth week after injecting cells, we anaesthetized the mice and photographed them in 


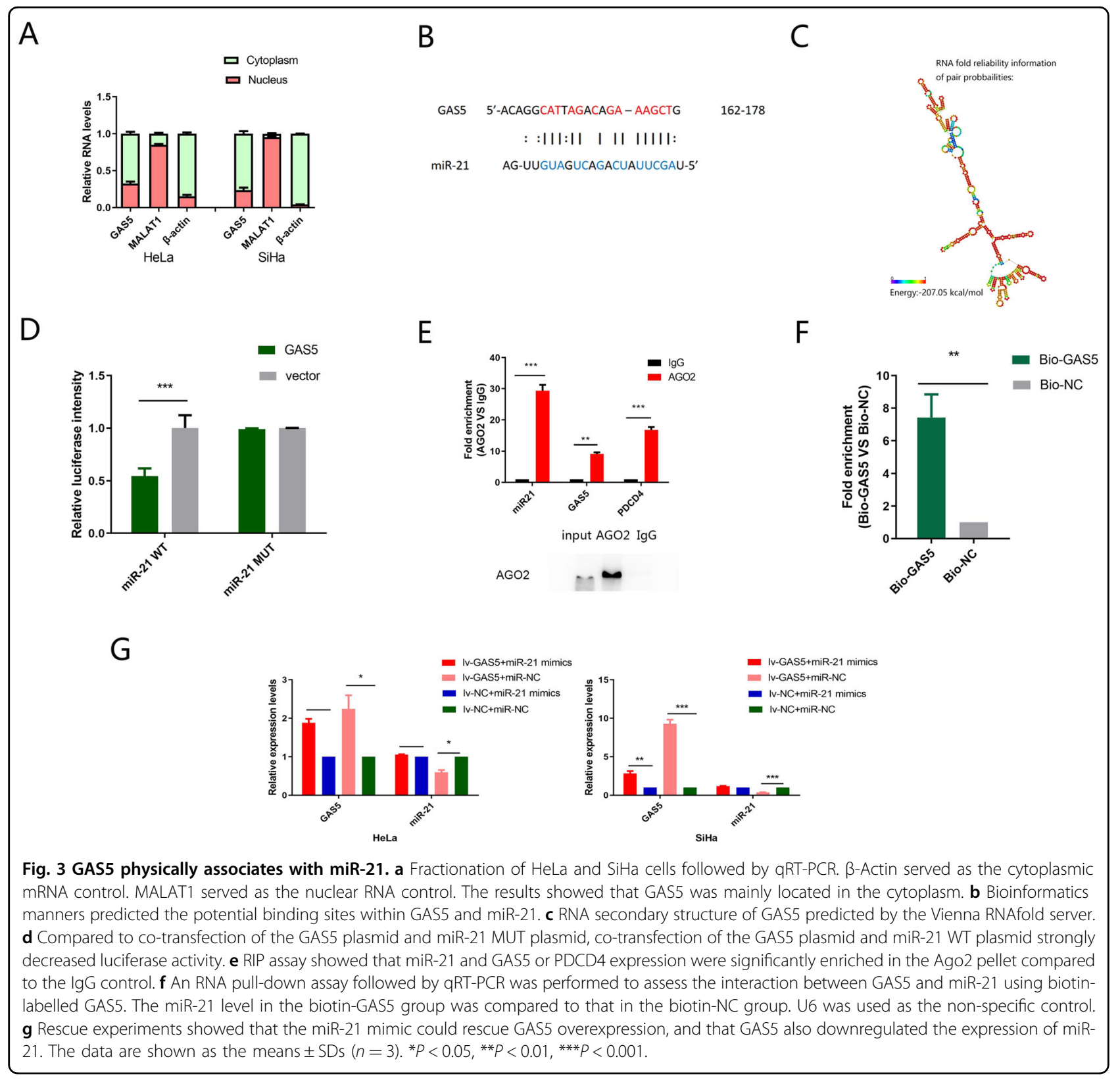

groups (Fig. 7b). Then, we injected cisplatin intraperitoneally once a week for 2 weeks to mimic chemotherapy in vivo. Tumour volumes were measured weekly until sacrifice. As expected, the tumour volumes in the lentivirus-GAS5 group were significantly smaller than those in the lentivirus-NC group. After injecting cisplatin, the tumour volume in the lentivirus-GAS5 group even showed a declining trend, while the tumour volume in the lentivirus-NC group increased (Fig. 7a). In sharp contrast, the tumours in the lentivirus-GAS5 group were invisible at the eighth week in vivo (Fig. 7c). Then, we sacrificed the mice and removed the tumours. A significant decrease in the tumour volume was observed macroscopically in the lentivirus-GAS5 group, and one of the tumours had even disappeared (Fig. 7d). Additionally, high PDCD4 expression was observed in the GAS5 overexpression group compared with the $\mathrm{NC}$ group as detected by IHC (Fig. 7e).

In summary, GAS5 significantly enhanced the cisplatin sensitivity of $\mathrm{CC}$ in vivo by upregulating PDCD4 expression.

\section{Discussion}

Cisplatin is one of the most widely used antineoplastic drugs for the treatment of numerous human cancers, including bladder, head and neck, lung, ovarian and 


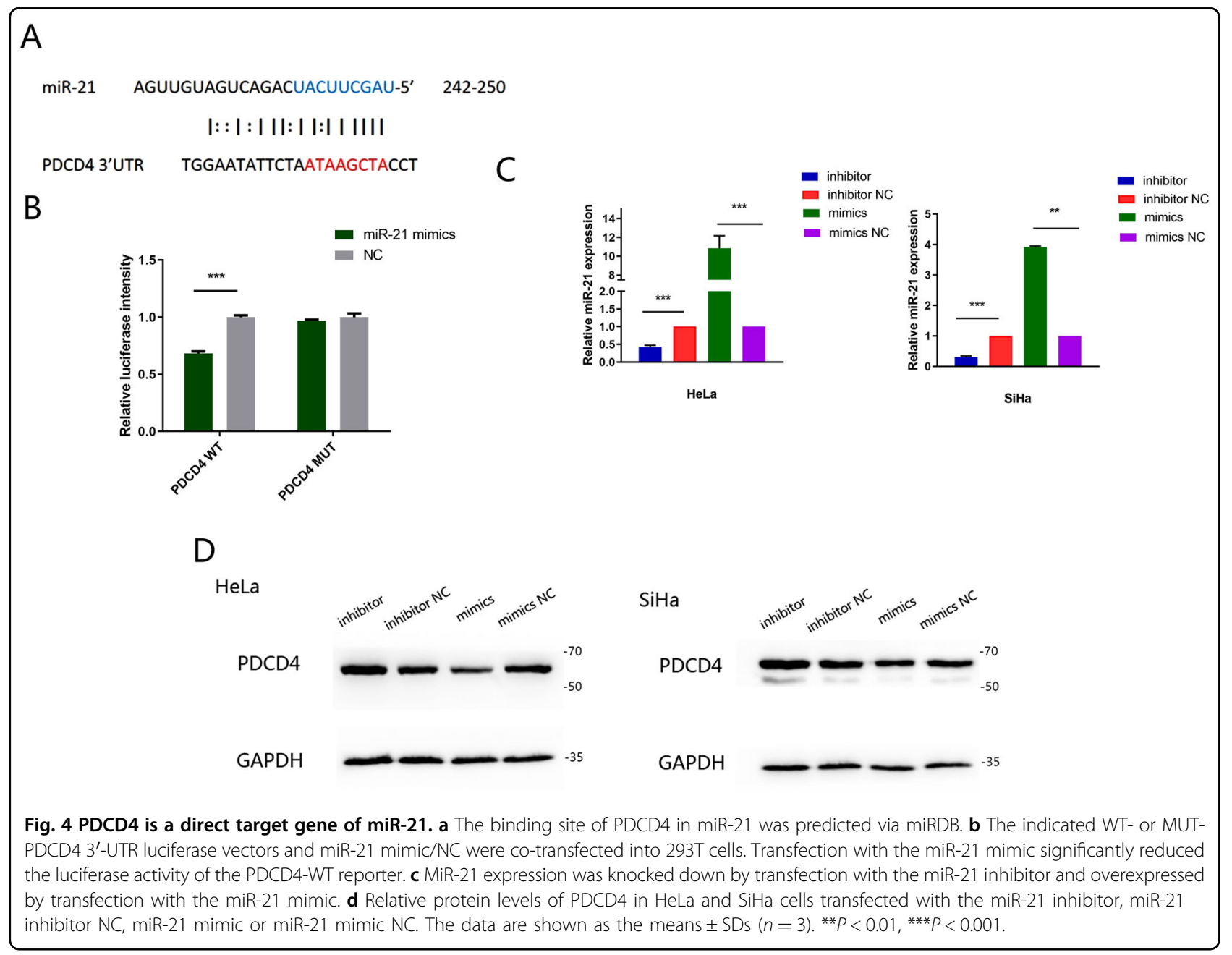

testicular cancers ${ }^{26,27}$. A major reason for the failure of cisplatin treatment is drug resistance ${ }^{28}$. Resistance to cisplatin, an important limiting factor affecting treatment efficacy, survival and prognosis, can easily lead to distant metastasis and relapse in $\mathrm{CC}^{29}$.

Numerous recent studies have examined the active molecular mechanisms during cancer progression and chemoresistance development. The discovery of lncRNAs provides a new entry point for us to study the mechanism of cisplatin resistance. Accumulating evidence has emphasized that lncRNAs possibly function as biomarkers and influence cancer chemoresistance ${ }^{30,31}$. LncRNA UCA1 could increase CDDP resistance and inhibit cell apoptosis in oral squamous cell carcinoma $(\mathrm{OSCC})^{32}$. LncRNA HOXD-AS1 enhanced the sensitivity of glioma to cisplatin by competitively binding to miR- $204^{33}$. GAS5 is a key tumour suppressor gene that has attracted much attention recent years, the content of GAS5 in different types of tumour tissues is lower than that in adjacent normal tissues, and it plays an important role in controlling tumour progression and prognosis, invasion and metastasis, apoptosis and radiotherapy tolerance ${ }^{34,35}$. In colorectal cancer tissue samples, the expression of GAS5 was significantly lower than that of adjacent normal tissues. The tumour volume of the GAS5 low-expression group was large. In addition, patients with low GAS5 expression showed a shorter survival time. Statistical analysis revealed that the expression level of GAS5 was an independent risk factor and prognostic factor for colorectal cancer ${ }^{36}$. Our study analysed clinical specimens and revealed that GAS5 was elevated in CC tissues compared to corresponding normal cervical tissues. Low GAS5 expression was associated with a younger age, vaginal recurrence or metastasis, chemotherapy and poorer OS in $\mathrm{CC}$ patients. Which suggested that lncRNA GAS5 may be a potential prognostic indicator of $\mathrm{CC}$ associated with chemotherapy.

We further explored the mechanisms of GAS5 on the sensitivity to chemotherapy. STATs, a family of latent cytoplasmic proteins, can transduce signals from cytokine and growth factor receptors to the nucleus. Activated STATs are phosphorylated on tyrosine residues and then 


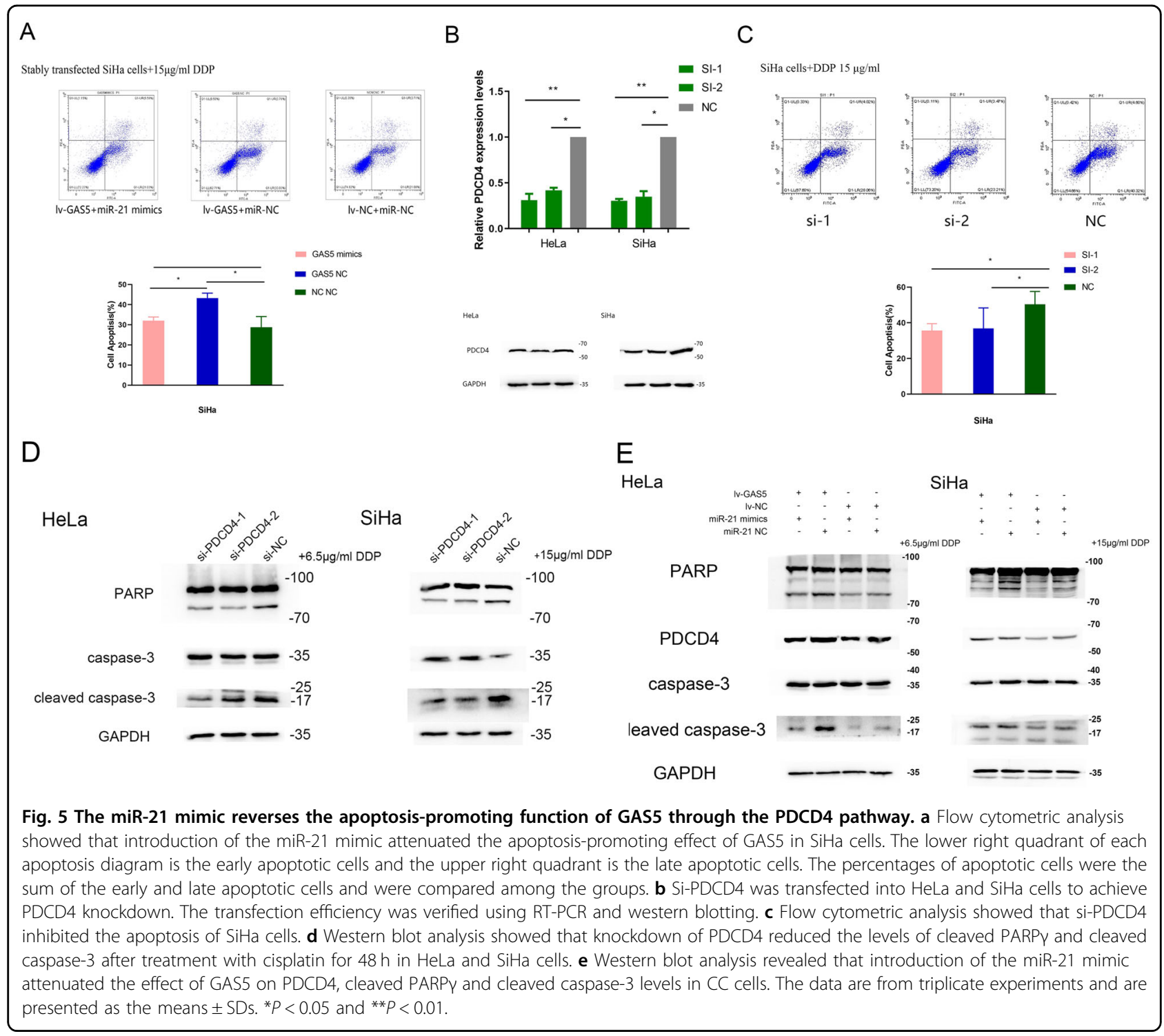

dimerize via reciprocal $\mathrm{SH} 2$ phosphotyrosine interactions and enter the nucleus to regulate the transcription of many different genes by binding to their promoter regions ${ }^{37}$. Persistent STAT activation in tumour cells abolishes the control of cell growth, survival, angiogenesis and immune function. To date, STAT3 is the most comprehensively explored STAT family member in terms of its contribution to cancer ${ }^{38}$. STAT3 signal is frequently activated during cancer development and associated with different characteristics of cancer. Research found that STAT3 was not activated in the periphery of non-tumour tissue and in the normal liver but was activated in hepatocellular carcinoma ${ }^{39}$. Recent studies verified that abnormal activation of STAT3 signalling mediates cisplatin sensitivity in several cancers, such as breast cancer, OSCC, oesophageal squamous cell carcinoma and ovarian cancer and lung cancer ${ }^{40-44}$. In CC, overexpression of
STAT3 was associated with cisplatin sensitivity ${ }^{45}$. Our study discovered that the phosphorylation levels of STAT3 in CC cells HeLa and SiHa were different from those in normal cervical squamous epithelial cells $\mathrm{H} 8$. Similarly, P-STAT3 was highly expressed in CC tissues compared to adjacent tissues. What is more, the expression of STAT3 in CC tissues was negatively correlated with the expression of GAS5. Therefore, we speculated that the role of GAS5 in the cisplatin sensitivity of CC was related to activation of STAT3. Via ChIP assay, we found that P-STAT3 could bind to the promoter region of GAS5. Validated that P-STAT3 is a transcription factor of GAS5. Additionally, further functional experiments demonstrated that inhibition of STAT3 phosphorylation promoted the expression of GAS5. Collectively, the above results suggested that P-STAT3 affected the transcription of GAS5 as a transcription factor. 


\section{A}

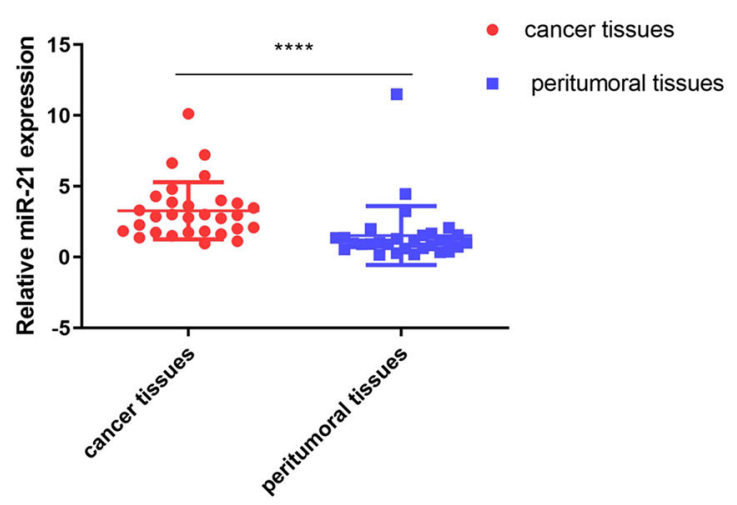

C

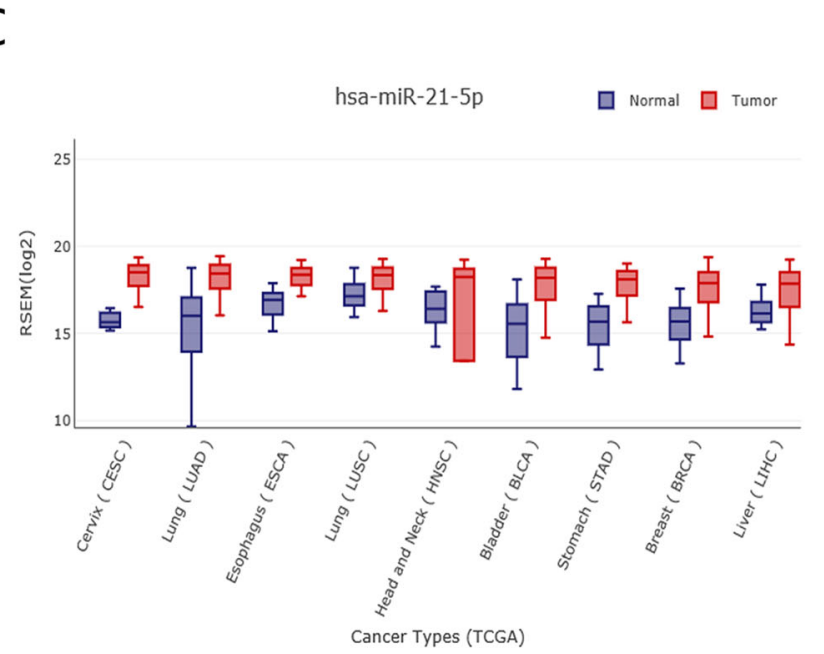

B

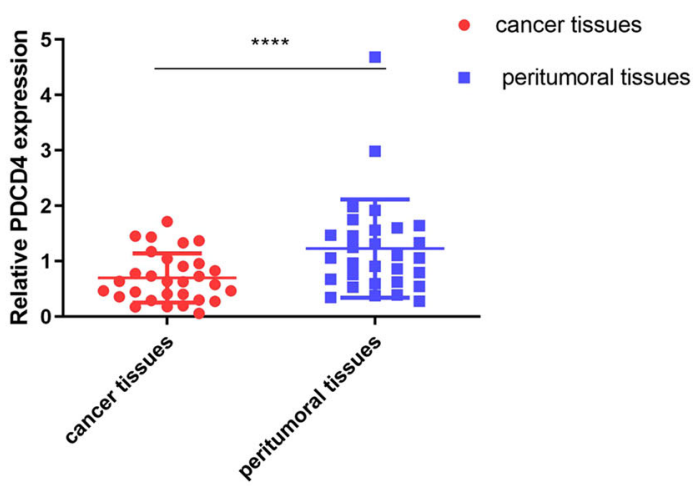

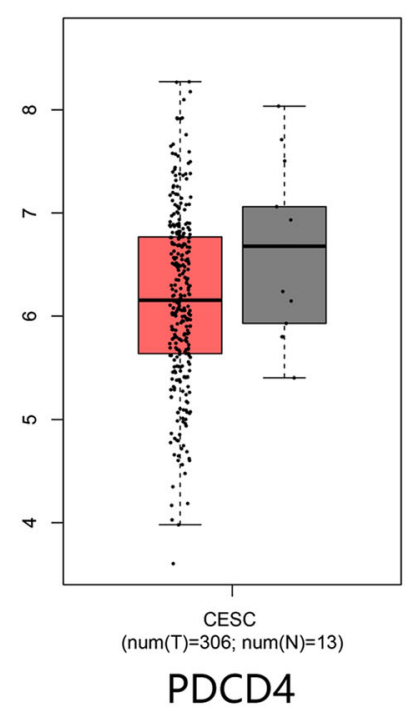

$\mathrm{D}$
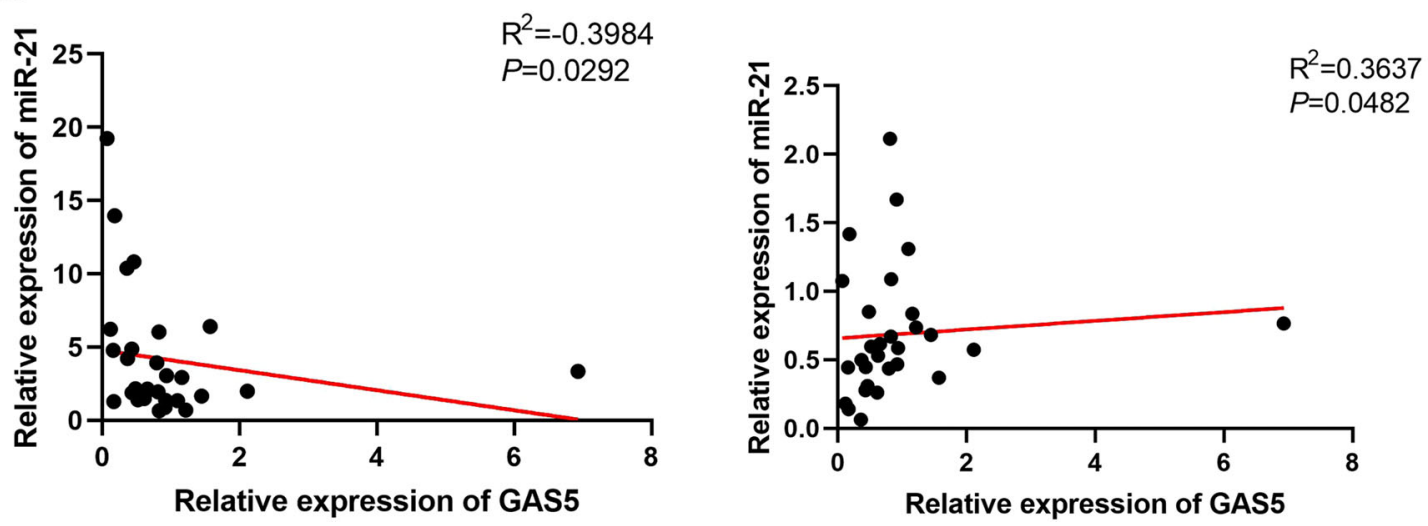

Fig. 6 PDCD4 expression in CC tissues and the connection between GAS5, miR-21 and PDCD4. a MiR-21 was highly expressed in tumour tissues compared to peritumoral tissues as determined by qPCR in cohort 1. b PDCD4 was expressed at low levels in tumour tissues compared to peritumoral tissues as detected by qPCR in cohort 1. c PDCD4 expression levels in CC tissues and paired non-tumour tissues from the TCGA database and miR-21 expression levels in CC tissues and paired non-tumour tissues in GEDS. $\mathbf{d}$ The Pearson correlations between GAS5 and miR-21 or GAS5 and PDCD4 were measured in 30 pairs of CC tissues. 


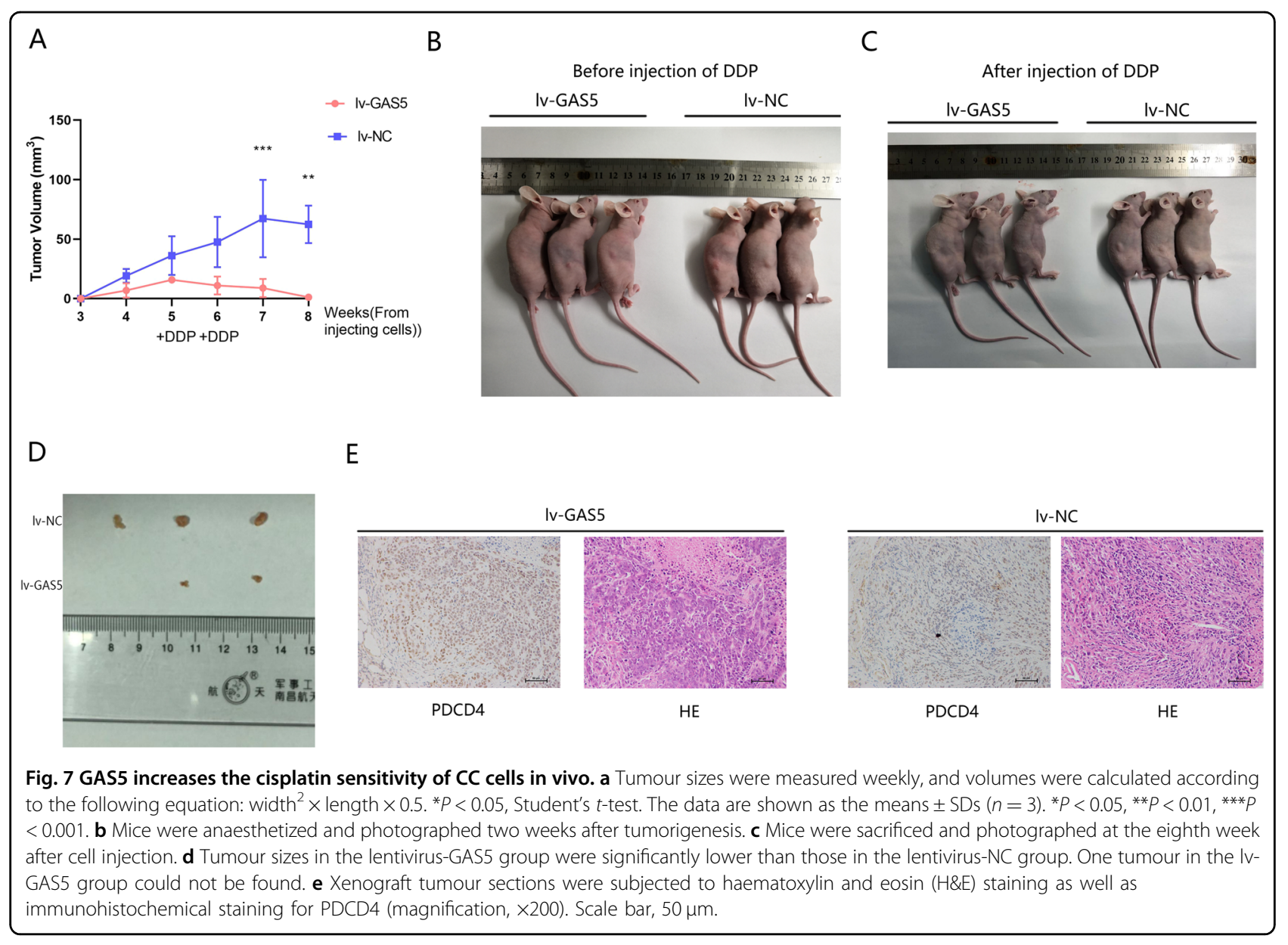

ceRNA is a transcript that can regulate each other at post-transcription level by competing miRNAs ${ }^{46}$. An increasing number of IncRNAs have been identified as ceRNAs that competitively bind with miRNAs to relieve translational repression of targeted mRNAs induced by common miRNAs and their downstream pathways ${ }^{47}$. When ceRNA, such as pseudogenes, is transcriptional silent, parent mRNAs are transcribed and exported to the cytoplasm, which is targeted by microRNA-guided RNAinduced silencing complex (miRNA-RISC) in the cytoplasm, leading to accelerated degradation, blocked translation and decreased expression. When ceRNA acquires transcriptional activity, it competes for miRNA targets, binds to RISC complexes, which results in the separation of miRNA-RISC complexes from parent genes and increased parental gene expression ${ }^{48}$. Miao et $\mathrm{al}^{49}$. reported that in ovarian cancer, ANRIL could interact with let-7a to further reduce HMGA2 levels, which promoted the apoptosis and improved the cisplatin sensitivity of ovarian cancer cells. In gastric cancer, IncRNA MT1JP regulated the expression of FBXW7 through competitively binding with miR-92a-3p, and played a role in inhibiting cell proliferation, migration, invasion and promoting cell apoptosis ${ }^{50}$. MiR-21 sponged by GAS5 has been reported in OSCC, hepatocellular carcinoma, laryngeal squamous cell carcinoma and ovarian cancer ${ }^{13,51-53}$. In our study, we verified the same mechanism in CC cells. QPCR analysis after transfection of the miR-21 mimic into cells with stable GAS5 overexpression revealed a negative dual regulatory relationship between GAS5 and miR-21. Furthermore, the results of the dual-luciferase reporter assay revealed that GAS5 contained the binding site with miR-21. RIP and RNA pull-down assays suggested that GAS5 formed an RISC complex with miR-21. Therefore, GAS5 inhibited the expression of miR-21 through "miRNA sponge".

PDCD4, which is originally found to participate in apoptosis, can selectively enhance chemosensitivity to platinum compounds mainly through the death receptormediated pathway ${ }^{54}$. Usually downregulated in tumours $^{55-57}$, PDCD4 can inhibit neoplastic transformation, control translation and induce apoptosis ${ }^{58}$. PDCD4 overexpression activated caspases $8,9,3$, lead to the apoptosis of liver cancer cells ${ }^{59}$. Our research identified PDCD4 as a target gene of miR-21, which played a key role in apoptosis. PDCD4 expression was higher in CC 


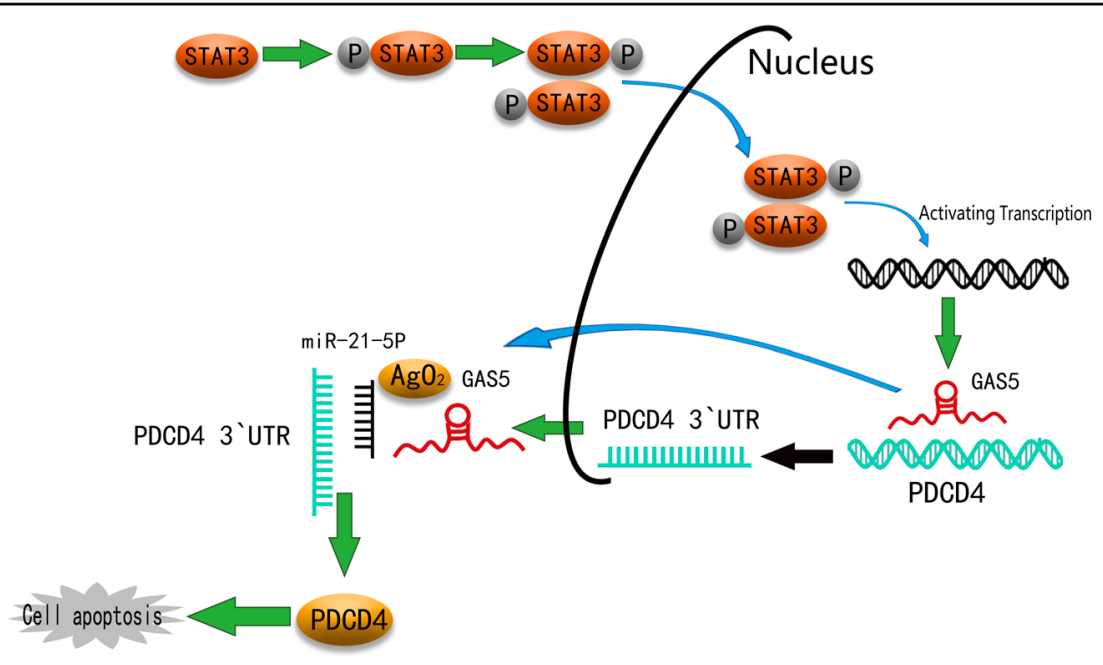

Fig. 8 Schematic diagram. GAS5, regulated by P-STAT3, enhances cisplatin sensitivity by acting as a ceRNA to regulate PDCD4 expression in CC.

tissues than in peritumoral tissues. Knockdown of PDCD4 restrained the apoptosis of CC cells. Therefore, we hypothesized that GAS5 promoted cisplatin-induced apoptosis by sponging miR-21 to indirectly regulate the expression of PDCD4. Ultimately, we observed that GAS5 promoted PDCD4 expression both in vitro and in vivo. In contrast, overexpression of miR-21 repressed the promoting effect of GAS5 overexpression on PDCD4 expression and cisplatin-induced apoptosis in CC cells. These data suggested that GAS5 promoted cisplatin sensitivity through the miR-21/PDCD4 axis.

Furthermore, we verified our conclusion in vivo. Research discovered that GAS5 overexpression could decrease the tumour growth, volume as well as weight of nude mice in vivo experiment ${ }^{60}$. Our study found that with cisplatin injection, the tumour volumes in the GAS5 overexpression group presented a declining trend, which was not due to the influence of proliferation but rather apoptosis, indicating that GAS5 overexpression enhanced the cisplatin sensitivity of xenograft tumours. Collectively, these findings indicate that GAS5 is a positive regulator of cisplatin sensitivity in $\mathrm{CC}$, and that targeting GAS5 may be an effective method for combating CC chemoresistance.

\section{Conclusions}

Overall, our data provided evidence that low GAS5 expression in CC tissues compared to normal tissues was connected with prognosis. Regulated by P-STAT3, GAS5 enhanced cisplatin sensitivity through the GAS5/miR-21/ PDCD4 pathway (Fig. 8). Because low GAS5 expression is more likely to occur in young women with $\mathrm{CC}$, therapeutics that target GAS5 may be a promising cisplatin- based chemotherapeutic strategy for use in younger patients.

\section{Web links and URLs \\ GEPIA (http://gepia.cancer-pku.cn/) \\ UCSC (http://genome.ucsc.edu/) \\ UCSC Xena (https://xenabrowser.net/heatmap/) \\ JASPAR CORE database (http://jaspar.genereg.net/) \\ ViennaRNA Web Services (http://rna.tbi.univie.ac.at/) \\ miRDB (http://mirdb.org/) \\ GEDS (http://bioinfo.life.hust.edu.cn/web/GEDS/)}

\section{Acknowledgements}

This work was supported by the National Natural Science Foundation of China (81572575), the Guangdong Province Natural Scientific Grant

(2016A020215059), the Special Support for Guangdong College Students' Innovation and Entrepreneurship Training Program (1055813194), the National College Students' Innovation and Entrepreneurship Training Program

(201310558097), and the Guangdong Clinical Teaching Base Teaching Program (2018JD004).

\section{Data availability}

All data that support the findings of this study are available from the corresponding authors upon reasonable request.

\section{Conflict of interest}

The authors declare that they have no conflict of interest.

\section{Publisher's note}

Springer Nature remains neutral with regard to jurisdictional claims in published maps and institutional affiliations.

Supplementary Information accompanies this paper at (https://doi.org/ 10.1038/s41419-020-2735-2).

Received: 24 February 2020 Revised: 21 June 2020 Accepted: 25 June 2020 Published online: 13 July 2020 


\section{References}

1. Siegel, R. L., Miller, K. D. \& Jemal, A. Cancer statistics, 2019. CA Cancer J. Clin. 69, 7-34 (2019).

2. Walboomers, J. M. et al. Human papillomavirus is a necessary cause of invasive cervical cancer worldwide. J. Pathol. 189, 12-19 (1999).

3. du Toit, G. C. \& Kidd, M. Prospective quality of life study of South African women undergoing treatment for advanced-stage cervical cancer. Clin. Ther. 37, 2324-2331 (2015)

4. Menderes, G., Black, J., Schwab, C. L. \& Santin, A. D. Immunotherapy and targeted therapy for cervical cancer: an update. Expert Rev. Anticancer Ther. 16 83-98 (2016).

5. Pfaendler, K. S., Liu, M. C. \& Tewari, K. S. Bevacizumab in cervical cancer: 5 years after. Cancer J. 24, 187-192 (2018).

6. Agoni, L. et al. Rigosertib is a more effective radiosensitizer than cisplatin in concurrent chemoradiation treatment of cervical carcinoma, in vitro and in vivo. Int. J. Radiat. Oncol. Biol. Phys. 88, 1180-1187 (2014).

7. Fabri, $\mathrm{V}$. A. et al. The impact of addition of consolidation chemotherapy to standard cisplatin-based chemoradiotherapy in uterine cervical cancer: matter of distant relapse. J. Oncol. 2019, 1217838 (2019).

8. Zhu, $\mathrm{H}$. et al. Molecular mechanisms of cisplatin resistance in cervical cancer. Drug Des. Dev. Ther. 10, 1885-1895 (2016).

9. Ghosh, S. Cisplatin: the first metal based anticancer drug. Bioorg. Chem. 88, 102925 (2019).

10. Di, C. \& Zhao, Y. Multiple drug resistance due to resistance to stem cells and stem cell treatment progress in cancer (Review). Exp. Ther. Med. 9, 289-293 (2015).

11. Abu, N., Hon, K. W., Jeyaraman, S. \& Jamal, R. Long noncoding RNAs as biotargets in cisplatin-based drug resistance. Future Oncol. 14, 3085-3095 (2018).

12. Rinn, J. L. \& Chang, H. Y. Genome regulation by long noncoding RNAs. Annu. Rev. Biochem. 81, 145-166 (2012).

13. Ma, N. et al. Long non-coding RNA GAS5 inhibits ovarian cancer cell proliferation via the control of microRNA-21 and SPRY2 expression. Exp. Ther. Med. 16, 73-82 (2018).

14. Yang, J., Hao, T., Sun, J., Wei, P. \& Zhang, H. Long noncoding RNA GAS5 modulates alpha-Solanine-induced radiosensitivity by negatively regulating miR-18a in human prostate cancer cells. Biomed. Pharmacother. 112, 108656 (2019).

15. Zhang, Z. et al. Negative regulation of IncRNA GAS5 by miR-21. Cell Death Differ. 20, 1558-1568 (2013).

16. Yao, T. et al. Growth arrest-specific 5 attenuates cisplatin-induced apoptosis in cervical cancer by regulating STAT3 signaling via miR-21. J. Cell. Physiol. 234 9605-9615 (2019).

17. Gao, J., Yin, X., Yu, X., Dai, C. \& Zhou, F. Long noncoding LINC01551 promotes hepatocellular carcinoma cell proliferation, migration, and invasion by acting as a competing endogenous RNA of microRNA-122-5p to regulate ADAM10 expression. J. Cell. Biochem. 120, 16393-16407 (2019).

18. King, V. M. \& Borchert, G. M. MicroRNA expression: protein participants in microRNA regulation. Methods Mol. Biol. 1617, 27-37 (2017).

19. Yao, T. \& Lin, Z. MiR-21 is involved in cervical squamous cell tumorigenesis and regulates CCL20. Biochim. Biophys. Acta 1822, 248-260 (2012).

20. Chen, D. et al. LncRNA growth arrest-specific transcript 5 targets miR-21 gene and regulates bladder cancer cell proliferation and apoptosis through PTEN. Cancer Med. 9, 2846-2858 (2020).

21. Meng, $\mathrm{H}$. et al. MicroRNA-330-3p functions as an oncogene in human esophageal cancer by targeting programmed cell death 4. Am. J. Cancer Res. 5, 1062-1075 (2015)

22. $\mathrm{Xu}, \mathrm{C}$. et al. TUG1 confers cisplatin resistance in esophageal squamous cell carcinoma by epigenetically suppressing PDCD4 expression via EZH2. Cell Biosci. 8, 61 (2018)

23. Yang, Q., Zhang, Z., Xu, H. \& Ma, C. Lidocaine alleviates cytotoxicity-resistance in lung cancer A549/DDP cells via down-regulation of miR-21. Mol. Cell. Biochem. 456, 63-72 (2019).

24. Zhen, Y. et al. miR-374a-CCND1-pPI3K/AKT-c-JUN feedback loop modulated by PDCD4 suppresses cell growth, metastasis, and sensitizes nasopharyngeal carcinoma to cisplatin. Oncogene 36, 275-285 (2017)

25. Yao, X. et al. Arctigenin enhances chemosensitivity of cancer cells to cisplatin through inhibition of the STAT3 signaling pathway. J. Cell. Biochem. 112 2837-2849 (2011)

26. Dasari, S. \& Tchounwou, P. B. Cisplatin in cancer therapy: molecular mechanisms of action. Eur. J. Pharmacol. 740, 364-378 (2014).
27. Voigt, W., Dietrich, A. \& Schmoll, H. J. [Overview of development status and clinical action. Cisplatin and its analogues]. Pharmazie unserer Zeit 35, 134-143 (2006).

28. Siddik, Z. H. Cisplatin: mode of cytotoxic action and molecular basis of resistance. Oncogene 22, 7265-7279 (2003).

29. Roy, M. \& Mukherjee, S. Reversal of resistance towards cisplatin by curcumin in cervical cancer cells. Asian Pac. J. Cancer Prev. 15, 1403-1410 (2014).

30. Dong, $\mathrm{H}$. et al. Activation of LnCRNA TINCR by H3K27 acetylation promotes Trastuzumab resistance and epithelial-mesenchymal transition by targeting MicroRNA-125b in breast cancer. Mol. Cancer 18, 3 (2019).

31. Wei, X. et al. Low expression of TUG1 promotes cisplatin sensitivity in cervical cancer by activating the MAPK pathway. J. BUON 24, 1020-1026 (2019).

32. Fang, Z. et al. LncRNA UCA1 promotes proliferation and cisplatin resistance of oral squamous cell carcinoma by suppressing miR-184 expression. Cancer Med. 6, 2897-2908 (2017)

33. Zhou, H., Ma, Y., Zhong, D. \& Yang, L. Knockdown of IncRNA HOXD-AS1 suppresses proliferation, migration and invasion and enhances cisplatin sensitivity of glioma cells by sponging miR-204. Biomed. Pharmacother. 112 108633 (2019).

34. Lorusso, D., Petrelli, F., Coinu, A., Raspagliesi, F. \& Barni, S. A systematic review comparing cisplatin and carboplatin plus paclitaxel-based chemotherapy for recurrent or metastatic cervical cancer. Gynecol. Oncol. 133, 117-123 (2014).

35. Mourtada-Maarabouni, M., Hedge, V. L., Kirkham, L., Farzaneh, F. \& Williams, G. T. Growth arrest in human T-cells is controlled by the noncoding RNA growth-arrest-specific transcript 5 (GAS5). J. Cell Sci. 121 939-946 (2008).

36. Yang, Y. et al. Long non-coding RNA GAS5 inhibits cell proliferation, induces G0/G1 arrest and apoptosis, and functions as a prognostic marker in colorectal cancer. Oncol. Lett. 13, 3151-3158 (2017).

37. Darnell, J. E. Jr. STATs and gene regulation. Science 277, 1630-1635 (1997).

38. $Y u, H$. \& Jove, R. The STATs of cancer-new molecular targets come of age. Nat. Rev. Cancer 4, 97-105 (2004).

39. Fathi, N., Rashidi, G., Khodadadi, A., Shahi, S. \& Sharifi, S. STAT3 and apoptosis challenges in cancer. Int. J. Biol. Macromol. 117, 993-1001 (2018).

40. Chang, M. T. et al. Chemosensitizing effect of honokiol in oral carcinoma stem cells via regulation of IL-6/Stat3 signaling. Environ. Toxicol. 33, 1105-1112 (2018).

41. Fang, Z., Chen, W., Yuan, Z., Liu, X. \& Jiang, H. LncRNA-MALAT1 contributes to the cisplatin-resistance of lung cancer by upregulating MRP1 and MDR1 via STAT3 activation. Biomed. Pharmacother. 101, 536-542 (2018).

42. $\mathrm{Xu}, \mathrm{H}$. et al. Tumor-derived mesenchymal-stem-cell-secreted IL-6 enhances resistance to cisplatin via the STAT3 pathway in breast cancer. Oncol. Lett. 15, 9142-9150 (2018)

43. Zhao, Y. et al. MicroRNA-125a-5p enhances the sensitivity of esophageal squamous cell carcinoma cells to cisplatin by suppressing the activation of the STAT3 signaling pathway. Int. J. Oncol. 53, 644-658 (2018).

44. Zou, M., Xu, C., Li, H., Zhang, X. \& Fan, W. 3,3'-Diindolylmethane suppresses ovarian cancer cell viability and metastasis and enhances chemotherapy sensitivity via STAT3 and Akt signaling in vitro and in vivo. Archiv. Biochem. Biophys. https://doi.org/10.1016/j.abb.2018.07.002 (2018).

45. Huang, L. L. \& Rao, W. SiRNA interfering STAT3 enhances DDP sensitivity in cervical cancer cells. Eur. Rev. Med. Pharmacol. Sci. 22, 4098-4106 (2018).

46. Qi, X. et al. ceRNA in cancer: possible functions and clinical implications. J. Med. Genet. 52, 710-718 (2015).

47. Tay, Y., Rinn, J. \& Pandolfi, P. P. The multilayered complexity of ceRNA crosstalk and competition. Nature 505, 344-352 (2014).

48. Thomson, D. W. \& Dinger, M. E. Endogenous microRNA sponges: evidence and controversy. Nat. Rev. Genet. 17, 272-283 (2016).

49. Miao, J. T. et al. LncRNA ANRIL affects the sensitivity of ovarian cancer to cisplatin via regulation of let-7a/HMGA2 axis. Biosci. Rep. 39, https://doi.org/ 10.1042/bsr20182101 (2019).

50. Zhang, G. et al. LncRNA MT1.JP functions as a ceRNA in regulating FBXW7 through competitively binding to miR-92a-3p in gastric cancer. Mol. Cancer 17, 87 https://doi.org/10.1186/s12943-018-0829-6 (2018).

51. $\mathrm{Hu}$, L. et al. Long noncoding RNA GAS5 suppresses the migration and invasion of hepatocellular carcinoma cells via miR-21. Tumour Biol. 37, 2691-2702 (2016).

52. Lyu, $\mathrm{K}$. et al. Long noncoding RNA GAS5 acts as a tumor suppressor in laryngeal squamous cell carcinoma via miR-21. Cancer Manag. Res. 11, 8487-8498 (2019) 
53. Zeng, B. et al. LnCRNA GAS5 suppresses proliferation, migration, invasion, and epithelial-mesenchymal transition in oral squamous cell carcinoma by regulating the miR-21/PTEN axis. Exp. Cell Res. 374, 365-373 (2019).

54. Zhang, $X$. et al. Programmed cell death 4 enhances chemosensitivity of ovarian cancer cells by activating death receptor pathway in vitro and in vivo. Cancer Sci. 101, 2163-2170 (2010).

55. Chen, Y. et al. Loss of PDCD4 expression in human lung cancer correlates with tumour progression and prognosis. J. Pathol. 200, 640-646 (2003).

56. Wang, D. et al. Programmed cell death factor 4 enhances the chemosensitivity of colorectal cancer cells to Taxol. Oncol. Lett. 18, 1402-1408 (2019).
57. Wei, N. A. et al. Loss of programmed cell death $4(\mathrm{Pdcd} 4)$ associates with the progression of ovarian cancer. Mol. Cancer 8, 70 (2009).

58. Matsuhashi, S., Manirujjaman, M., Hamajima, H. \& Ozaki, I. Control mechanisms of the tumor suppressor PDCD4: expression and functions. Int. J. Mol. Sci. 20 https://doi.org/10.3390/ijms20092304 (2019).

59. Zhang, $\mathrm{H}$. et al. Involvement of programmed cell death 4 in transforming growth factor-beta1-induced apoptosis in human hepatocellular carcinoma. Oncogene 25, 6101-6112 (2006).

60. Yang, W. et al. Upregulation of IncRNA GAS5 inhibits the growth and metastasis of cervical cancer cells. J. Cell. Physiol. 234, 23571-23580 (2019). 